\title{
Outer Diameter
}

National Cancer Institute

\section{Source}

National Cancer Institute. Outer Diameter. NCI Thesaurus. Code C124136.

The longest possible length of a straight line passing through the center of a circular or spheroid object that connects two points on the outer circumference. 